\title{
Gust Response Decomposition in a Stator/Rotor Axial Compressor with Varying Axial Gap
}

\author{
Andrew M. Wo,* Meng-Hsuan Chung, $\dagger$ and Shu-Tzung Hsuł \\ National Taiwan University, Taipei 106, Taiwan, Republic of China
}

\begin{abstract}
The objective of this paper is to decompose the unsteady force on the rotor of a stator/rotor axial compressor into vortical and potential contributions, for axial gaps of 10,20 , and $30 \%$ chord between blade rows. Three methods of decomposition are proposed. The final method adopted requires two steps. First, the potential contributed gust within the gap region is found using a panel code for stator/rotor configuration and the vortical contributed gust from the difference between that calculated by a Navier - Stokes code and the potential gust. Second, the rotor gust response is decomposed using the NavierStokes code in the rotor cascade configuration, with the vortical and potential disturbances as separate inlet boundary conditions. Results show that the rotor gust response caused by vortical contribution is dominant when the upstream wake impinges upon the rotor leading edge. The rotor gust response caused by potential contribution reaches an extremum when the stator trailing edge is closest to the rotor leading edge. Both vortical and potential contributions are important to determine the total blade response when the axial gap is less than about $30 \%$ of the chord.
\end{abstract}

\begin{tabular}{|c|c|}
\hline \multicolumn{2}{|r|}{ Nomenclature } \\
\hline$C$ & $=$ chord \\
\hline$C_{p}$ & $=$ static pressure coefficient \\
\hline$C_{1}, C_{2}, C_{\mu}$ & $=$ closure coefficients of turbulence model \\
\hline$D$ & $\begin{aligned}= & \text { additional dissipation term of turbulence } \\
& \text { kinetic energy }\end{aligned}$ \\
\hline$E, F$ & $\begin{array}{l}=\text { production and dissipation term of turbulence } \\
\text { dissipation rate, respectively }\end{array}$ \\
\hline$f_{\mu}, f_{\mu_{1}}, f_{\mu_{2}}$ & $=$ closure coefficients of turbulence model \\
\hline & $=$ production term of turbulence kinetic energy \\
\hline$h$ & $\begin{aligned}= & \text { circumferential spacing of line vortices in } \\
& \text { panel method }\end{aligned}$ \\
\hline$i, i$ & $=$ unit vector in axial direction, $\sqrt{-1}$ \\
\hline$j$ & $=$ unit vector in tangential direction \\
\hline$k$ & $=$ turbulence kinetic energy \\
\hline$p$ & $=$ static pressure \\
\hline $\operatorname{Re}$ & $\begin{aligned}= & \text { Reynolds number based on inlet flow velocity } \\
& \text { and blade chord }\end{aligned}$ \\
\hline$R_{t}$ & $=$ turbulence Reynolds number \\
\hline$S$ & $=$ total length of a blade surface \\
\hline$s$ & $=$ coordinate along blade surface \\
\hline$T$ & $=$ blade-to-blade period or spanwise moment \\
\hline$t$ & $=$ time \\
\hline$U_{\tau}$ & $=$ wall shear velocity \\
\hline$U_{\infty}$ & $=$ inlet uniform velocity \\
\hline$u$ & $\begin{aligned}= & \text { streamwise component of instantaneous } \\
& \text { velocity }\end{aligned}$ \\
\hline $\boldsymbol{u}$ & $=$ absolute flow velocity vector, $u \boldsymbol{i}+v \boldsymbol{j}$ \\
\hline $\boldsymbol{u}_{b}$ & $=$ rotor blade wheel velocity vector \\
\hline$V$ & $=$ magnitude of instantaneous absolute velocity \\
\hline$v$ & $=$ transverse component of instantaneous velocity \\
\hline$W$ & $\begin{array}{l}=\text { periodic unsteady relative velocity from NS } \\
\text { code }\end{array}$ \\
\hline
\end{tabular}

Received Dec. 16, 1995; revision received Aug. 20, 1996; accepted for publication Sept. 16, 1996. Copyright (c) 1996 by the authors. Published by the American Institute of Aeronautics and Astronautics, Inc., with permission.

*Associate Professor, Institute of Applied Mechanics. E-mail: andrew@ spring.iam.ntu.edu.tw.

$\dagger$ Associate Research Scientist, CFD and Environmental Engineering Section; currently Associate Research Scientist, National Center for High-Performance Computing, Hsinchu 300, Taiwan, ROC.

$\ddagger$ Doctoral Student.

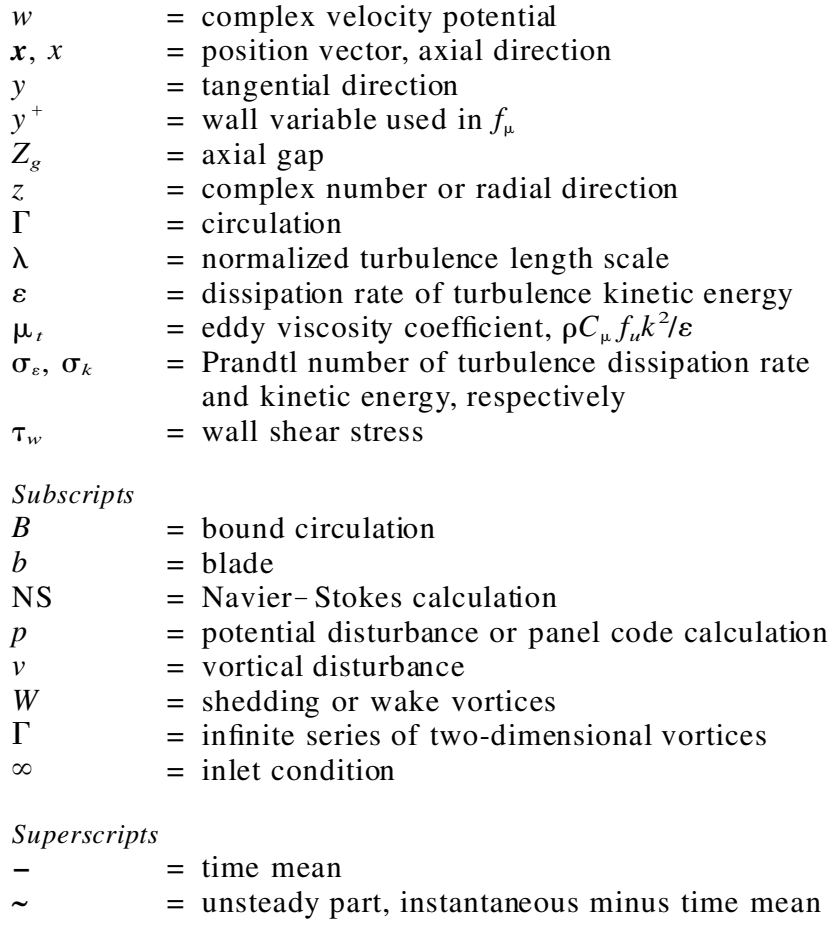

\section{Introduction}

T HE unsteady force caused by forced response on a turbomachinery blade is primarily because of flow unsteadiness in the vicinity of the blade, which includes passing of wakes, the potential field from the upstream blade row, the potential effect from the downstream row, and all other timevarying flow features. Disastrous blade failure can occur when the flow excitation matches the blade's natural frequency.

Many researchers have undertaken studies of gust and gust response in axial turbomachines. An excellent review of the subject can be found by AGARD. ${ }^{1}$ Kielb and Chiang ${ }^{2}$ provided a summary of recent advances in forced response analyses. Verdon $^{3}$ also reviewed unsteady aerodynamic methods for turbomachinery aeroelastic and aeroacoustic applications.

Significant progress in flow modeling has been made in recent years. Verdon and $\mathrm{Hall}^{4}$ developed a two-dimensional lin- 
earized flow analysis with two different gust-modeling capabilities: one termed frozen gust, in which the gust does not interact with the mean flowfield and remains constant through an airfoil row, and the second termed distorted gust, in which the gust interacts with the mean flow and changes the amplitude and shape in the process. Hall and Crawley ${ }^{5}$ used a twodimensional linearized Euler analysis to predict unsteady pressures caused by gusts. Giles ${ }^{6}$ calculated wake/rotor interaction using nonlinear Euler analyses in two-dimensional flows. A three-dimensional unsteady Navier-Stokes solver was developed by Rai ${ }^{7}$ and Rai and Madavan. ${ }^{8}$ Manwaring and Wisler ${ }^{9}$ made a substantial contribution in comparing current state-ofthe-art gust response analyses with experimental data. They showed that an approach in which the unsteady gust is linearized about the time-mean nonlinear flow is appropriate. Among other conclusions, they highlighted the importance of properly accounting for both vortical and potential disturbances in predicting gust response. Henderson and Fleeter ${ }^{10}$ identified the importance of pressure disturbance by showing that airfoil wakes are not able to be modeled by the boundary conditions of the current state-of-the-art linear vortical gust unsteady aerodynamic theory. They also quantitatively showed that the type of forcing function used significantly affects the resulting gust response, with the complexity of the response characteristics increasing from the perforated plate to the airfoil cascade forcing functions. ${ }^{11}$ Hence, modeling the static pressure fluctuations as arising from the rotor airfoil potential field and incorporating the rotor potential into the analysis may improve the data-theory correlation. ${ }^{10}$ Chung and $\mathrm{Wo}^{12}$ proposed a method of decomposing the flow unsteadiness into vortical and potential contributions using Navier-Stokes and panel codes, which, in essence, include both velocity and pressure disturbances (more details are provided in Sec. III).

The goal of this paper is to decompose the rotor gust response into vortical and potential contributions, for various axial gaps between stator/rotor blade rows, and to study their individual effect on the total unsteady loading during a bladeto-blade cycle.

\section{Numerical Methods}

\section{A. Navier-Stokes Calculations}

Calculations are performed using a two-dimensional incompressible Reynolds-averaged Navier-Stokes code written for multistage configurations. Numerical details and validations are presented by Chung and Wo ${ }^{12}$ and Chung. ${ }^{13}$

The two-dimensional Reynolds-averaged Navier-Stokes equations for incompressible flow are used in this work. Governing equations in the blade relative frame are

$$
\begin{gathered}
\nabla \cdot \boldsymbol{u}=0 \\
\frac{\partial \boldsymbol{u}}{\partial t}+\nabla \cdot\left[\left(\boldsymbol{u}-\boldsymbol{u}_{b}\right) \boldsymbol{u}\right]=-\nabla P+\nabla \cdot\left[\left(\mu+\mu_{t}\right) \nabla \boldsymbol{u}\right]
\end{gathered}
$$

The modified Launder-Sharma low-Re version of the $k-\varepsilon$ twoequation model $^{14}$ is used to close the previous equations via the eddy viscosity coefficient $\mu_{t}$. The governing equations used are

$$
\begin{aligned}
& \frac{\partial k}{\partial t}+\nabla \cdot\left[\left(\boldsymbol{u}-\boldsymbol{u}_{b}\right) k\right]=\nabla \cdot\left[\left(\mu+\frac{\mu_{t}}{\sigma_{k}}\right) \nabla k\right] \\
& +G_{k}-\varepsilon-D \\
& \frac{\partial \varepsilon}{\partial t}+\nabla \cdot\left[\left(\boldsymbol{u}-\boldsymbol{u}_{b}\right) \varepsilon\right]=\nabla \cdot\left[\left(\mu+\frac{\mu_{t}}{\sigma_{\varepsilon}}\right) \nabla \varepsilon\right] \\
& \quad+C_{1} f_{\mu_{1}} G_{k} \frac{\varepsilon}{k}-C_{2} f_{\mu_{2}} \frac{\varepsilon^{2}}{k}+E-F
\end{aligned}
$$

where

$$
\begin{aligned}
& G_{k}=\mu_{t}\left(\frac{\partial u_{i}}{\partial x_{k}}\right)\left(\frac{\partial u_{i}}{\partial x_{k}}+\frac{\partial u_{k}}{\partial x_{i}}\right), \quad D=2 \mu\left(\frac{\partial \sqrt{k}}{\partial x_{j}}\right)^{2} \\
& E=2 \mu \mu_{t}\left(\frac{\partial^{2} u_{i}}{\partial x_{j}^{2}}\right)^{2}, \quad F=2 \mu\left(\frac{\partial \sqrt{\varepsilon}}{\partial x_{j}}\right)^{2}, \quad C_{\mu}=0.09 \\
& C_{1}=1.44, \quad C_{2}=1.92, \quad \sigma_{k}=1.0, \quad \sigma_{\varepsilon}=1.22 \\
& f_{\mu}=\left[1-\exp \left(\frac{-y^{+}}{A^{+}}\right)\right]^{2}, \quad A^{+}=25, \quad f_{\mu_{1}}=1.0 \\
& f_{\mu_{2}}=1-0.21875 \exp \left(\frac{-R_{t}^{2}}{36}\right)
\end{aligned}
$$

with $R_{t}=\rho k^{2} /(\mu \varepsilon), y^{+}=\rho y U_{\tau} / \mu$, and $U_{\tau}=\sqrt{\tau_{w} / \rho}$.

Equations $(1-4)$, in their conservative form as shown, are solved by a finite volume approach using an iterative semiimplicit method for pressure-linked equations ${ }^{15}$ (SIMPLE). In this work, a nonstaggered grid is used with the computational nodes located at the grid cell center. For time discretization, a Crank-Nicolson-like scheme is used to achieve second-order time accuracy. For space discretization, the convection term is approximated by the QUICK scheme, ${ }^{16}$ whereas all other terms use a central difference scheme. The spatial differences are formally second-order accurate.

The computational domain is bounded by various boundaries including inflow/outflow boundaries (located $1.5 \mathrm{C}$ upstream of the stator leading edge and $1.5 C$ downstream of the rotor trailing edge, respectively), blade row interface boundaries, periodic boundaries, and rigid blade surfaces, as shown in Fig. 1. In this work, each blade row is discretized using a body-fitted embedded H-type grid. The main reason that the conventional pinched $\mathrm{H}$-type grid is not used is to avoid an extremely skewed mesh near the blade leading edge. To verify grid independence, two grid sizes were computed ${ }^{13}$ : 1) $172 \times$ 78 cells per row with 108 cells per blade surface and 2) 258 $\times 114$ cells per row with 160 cells per blade surface. The coarse grid was found to be satisfactory and is adopted throughout this work.

A slip interface is located at the midgap between the two blade rows, which separates two grid systems fixed with respect to each blade. Numerics are passed through the interface as provided in detail by Chung and $\mathrm{Wo}^{12}$ and Chung. ${ }^{13}$

Computation is performed using the in-house Cray YMP/EL with four processors. Typical calculation for stator/rotor configuration requires $3 \times 10^{-4} \mathrm{CPU}$ second per iteration per cell, with approximately 10 iterations per time step. About 20-30 blade-to-blade periods are required from impulsive start to periodic steady state. Thus, the total computational time is about 120 CPU hours.

Comparison of the unsteady force on the stator, of a rotor/ stator stage with an axial gap of $30 \%$ chord, between experiment and calculation, is shown in Fig. 2. The data were taken from an in-house, large-scale, low-speed, compressor rig with 10 surface-mounted Kulites per stator surface. The overall comparison is considered acceptable. However, the amplitude and fine features are not as well predicted. The issue of turbulence modeling is believed to be accountable for the discrepancy, since the unsteady force is dominated by wake impingement and subsequent wake convection within the blade passage for an axial gap of $30 \%$ chord.

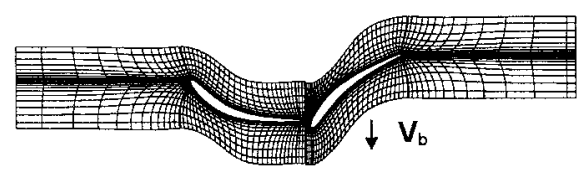

Fig. 1 Computational grid for the stator/rotor configuration (one-quarter density shown for clarity). 
a)

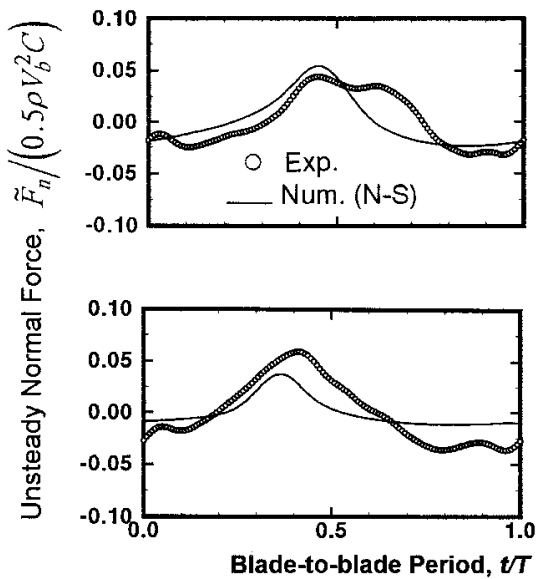

Fig. 2 Comparison of the calculated unsteady force, in the direction normal to the chord, on the stator of a rotor/stator configuration with data from surface-mounted Kulites pressure transducers: a) high loading and b) design loading.

\section{B. Panel Method Calculations}

The panel method solves for the velocity potential via $\nabla^{2} \phi$ $=0$. The boundary condition on the blade surface is $\partial \phi / \partial n=$ $\boldsymbol{u}_{b} \cdot \boldsymbol{n}$. Also, the circulation around a closed material loop remains unchanged, i.e., Kelvin's theorem, $D \Gamma / D t=0$. Finally, the flow must be smooth at the blade trailing edge, i.e., satisfy the Kutta condition that the velocity is finite at the trailing edge. The static pressure is found using the unsteady Bernoulli equation:

$$
\frac{P}{\rho}=\frac{p_{\infty}}{\rho}-\frac{\partial \phi}{\partial t}+\frac{1}{2}\left(U_{\infty}^{2}-v^{2}\right)
$$

A vortex panel method is used to solve this unsteady potential flowfield. A vorticity distribution of linearly varying strength is located on each panel. Wake vortices are shed from the blade trailing edge to satisfy Kelvin's theorem at each instant and the vorticity strength at the trailing edge is forced to zero to satisfy the Kutta condition. The approach used here is similar to that provided by Kuethe and Chow. ${ }^{17}$

To model the effects of blades in cascade, consider an infinite number of two-dimensional vortices with the strength $\Gamma$ on each vortex being constant and separated by a distance $h$. The complex velocity potential resulting from these vortices is ${ }^{18}$

$$
w_{\Gamma}(z)=i \Gamma \ln \left[\sin \frac{-i \pi\left(z-z_{\Gamma}\right)}{h}\right]
$$

with each vortex located at $z_{\Gamma}+i n h$, where $\eta$ is an integer. From the relationship between the complex velocity and physical velocity, $\mathrm{d} w / \mathrm{d} z=u-i v$, the induced velocity in the flow can be found. The manner in which vortices are shed into the flow, to conserve Kelvin's theorem, is based on the criterion by Basu and Hancock. ${ }^{19}$ A total of 201 panels are used on each blade. The interblade phase angle is set to zero, since it is assumed that there is no phase angle between any pair of adjacent blades in the process of vortex shedding and the blades have no vibratory motion.

Numerical difficulty exists when a shed vortex is near a panel surface or even penetrating it. To avoid nonphysical velocity, if the normal distance between the vortex and the surface panel is less than $1 \%$ of $h / \pi$, the calculated velocity is set to that of the nearest panel control point. If a vortex is penetrating a panel, a fully elastic collision is assumed, which relocates the vortex as rebounded from the panel surface.

To reduce computing cost as a result of a large number of vortices in the flow, a cutoff plane is located at three chord

lengths downstream of the trailing edge of the rotor blade row. Vortices of the same sign beyond that plane are merged to one vortex every 10 time steps. Its position and strength are such that the total circulation and the first integral moment of the vorticity distribution outside the cutoff plane are both conserved.

Computation is performed in a DEC scalar workstation. A typical calculation for a stator/rotor configuration requires about $6 \mathrm{CPU}$ hours from impulsive start to periodic steady state, corresponding to 12 blade-to-blade periods.

\section{Gust Decomposition}

The decomposition of gust in the gap region uses both the Navier-Stokes and potential codes. The key concept here is that the potential disturbance, as defined next, calculated by the potential code, represents the potential disturbance included in the Navier-Stokes results, i.e.,

$$
\tilde{\boldsymbol{u}}_{\mathrm{NS}}=\tilde{\boldsymbol{u}}_{v}+\tilde{\boldsymbol{u}}_{p}
$$

where $\tilde{\boldsymbol{u}}_{\mathrm{NS}}$ is the gust calculated by the Navier-Stokes code, $\tilde{\boldsymbol{u}}_{p}$ is the potential gust from the potential code, and $\tilde{\boldsymbol{u}}_{v}$ is the vortical gust from the difference between $\tilde{\boldsymbol{u}}_{\mathrm{NS}}$ and $\tilde{\boldsymbol{u}}_{p}$. Figure 3 illustrates this graphically. It is important to note that all disturbances are treated as being normal and parallel to the local time mean relative velocity vector as computed by the $\mathrm{Na}$ vier-Stokes code.

The manner in which the potential $\tilde{\boldsymbol{u}}_{p}$ is extracted from the potential code does not include contributions from shed vortices. Of course, in the calculation procedure, vortices are shed as dictated by Kelvin's theorem. The primary reason for excluding contributions from shed vortices is that, in a real fluid, shed vortices have their origin as a result of viscous phenomenon; they enter the flowfield via the boundary layer, which results in the wake being unsteady. ${ }^{20}$ Moreover, for a stator/ rotor unsteady calculation, if the gust includes contributions from shed vortices, this potential gust would persist far downstream since there is no mechanism for the shed vortices to decay, which is not physical. Thus, shed vortices are included in the vortical disturbance.

Feiereisen et al. ${ }^{21}$ proposed a disturbance-splitting method in which the contribution of the unsteady static pressure is incorporated through some weighting factors. Then the vortical and potential disturbance amplitudes are overdetermined and solved by developing a least-squares method. Comparing the complete and reconstructed gusts for the perforated plate and the airfoil cascade wake generators, they concluded that for a more appropriate gust-splitting method, it should incorporate not only velocity data, but also unsteady static pressure data. Their formulation recovers Giles' analysis ${ }^{6}$ (Appendix II of Manwaring and Wisler'), or Method V in the original paper, when the static pressure weighting factor is set to zero.

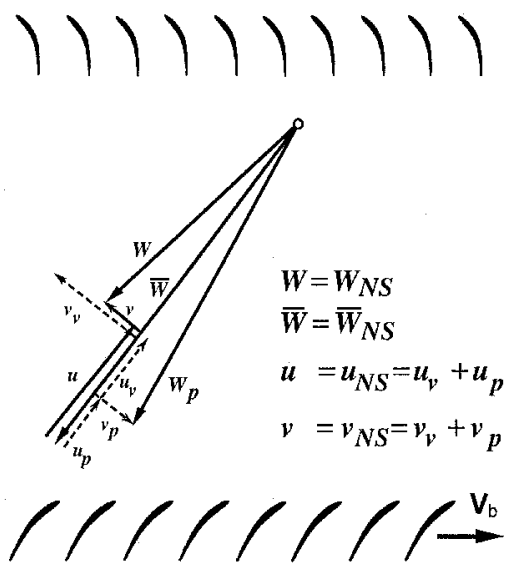

Fig. 3 Decomposition of vortical and potential gusts using Navier-Stokes and potential codes. 
The present gust-splitting method was compared with the analysis of Feiereisen et al. ${ }^{21}$ by Chung and Wo, ${ }^{12}$ with more details provided by Chung, ${ }^{13}$ which was proved to be physically sound. Although the present method only decomposes the velocity disturbance, both velocity and pressure disturbances are taken into account in calculating the gust response on a blade, since a Navier-Stokes code is used. Numerical details on this point are provided in Sec. IV.C.

\section{Gust Response Decomposition}

Three methods were examined to split the Navier-Stokes calculated gust response into vortical and potential components, i.e.,

$$
\tilde{P}_{\mathrm{NS}}=\tilde{P}_{v}+\tilde{P}_{p}
$$

where $P$ denotes the blade surface pressure. The following sections outline these methods and describe their particular features. Method 3 is finally adopted in this work.

\section{A. Control Volume Approach (Method 1)}

Consider a control volume moving with the rotor blade and bounded by the interfacial, periodic, suction, and pressure blade surface boundaries. For this control volume, one can derive an integral momentum equation that relates the instantaneous velocity and force, based on the Reynolds transport theorem. ${ }^{13}$ Taking a suitable time mean of this equation and subtracting it from the instantaneous equation results in an equation governing unsteady quantities, where the nonlinear gust can be linearized about the time mean. To decompose the linearized equation into vortical and potential parts, split the unsteady velocity into vortical and potential contributions and substitute it into the linearized equation. To calculate the response caused by an individual contribution, apply each decomposed equation in the circumferential direction, neglecting the shear force (proved to be reasonable by Navier-Stokes calculations); thus, the decomposed unsteady pressure force on the suction and pressure blade surfaces are related to the unsteady flow disturbance associated with the control volume.

The difficulty in applying this method lies in the calculation of the unsteady potential disturbance near the blade surface, where it changes drastically and unphysically because of the approaching shed vortices, which, in reality, should be sufficiently diffused before reaching the blade surface. Thus, this method, though physically sound, is abandoned because of numerical difficulties.

\section{B. Splitting Based on Calculated Response from Panel Code (Method 2)}

The second method attempted is intuitive. Using the unsteady Bernoulli's equation, the response caused by potential contribution is calculated directly from the panel code. The response caused by vortical contribution is obtained by subtracting the potential component from the Navier-Stokes calculated response. This method is also tamed with the same numerical trouble caused by shed vortices near the blade surface, as that of Method 1.3

\section{Splitting Based on Navier-Stokes Calculated Response (Method 3)}

To overcome the previous difficulty, the rotor blade response is calculated by the Navier-Stokes code in the rotor cascade configuration with either vortical or potential disturbances in the gap region as separate boundary conditions. The calculated response with the potential disturbance, plus the time mean, as the inlet boundary condition is the gust response caused by potential contribution, or simply, potential gust response. The gust response caused by vortical contribution, or simply vortical gust response, is found by subtracting the potential gust response from the Navier-Stokes calculated response for the stator/rotor configuration. With the gust response caused by both vortical and potential disturbances calculated from the same Navier-Stokes code, a consistent treatment in decomposing both the gust and the gust response is formed.

Both velocity and pressure disturbances are, in essence, taken into account when calculating the rotor gust response. For the potential gust response, the potential gust enters the problem as a direct boundary condition at the inlet, as previously mentioned. The potential contributed pressure disturbance is included via the nature of the Navier-Stokes calculation. At the inlet boundary condition of a rotor cascade configuration, the term $\partial p / \partial x$ is calculated using the potential gust from the panel code, where the term $\partial p / \partial y$ is not needed as a boundary condition. Since the gust and pressure disturbances resulting from potential contribution are governed by the unsteady Bernoulli equation, which is just a simplified form of the Navier-Stokes equation, the pressure disturbance is included automatically. This approach has an advantage of a uniquely determined system of governing equations and boundary conditions, while incorporating all physics involved.

In the rotor cascade calculation, the grid is exactly that used in the stator/rotor calculations to avoid the effect of having two grid arrangements. Because the QUICK scheme is used, the nodal values of flow variables, except the pressure on the exterior two-column grid adjacent to the upstream interface, must be given as the boundary condition. The values of velocity and mass flux, both vortical and potential, are obtained from the corresponding stator/rotor calculation and the gust decomposition method described previously. All other variables needed in the calculation, e.g., $k$ and $\varepsilon$, are the same as those calculated in the corresponding stator/rotor interaction.

\section{Compressor Parameters}

The blades used in the calculation are that of a low-speed three-repeating-stage axial compressor. The Reynolds number used in the computations is $1.59 \times 10^{5}$, based on the inlet axial velocity, or $2.91 \times 10^{5}$, based on the rotor inlet circumferential-averaged relative velocity, which corresponds to the near-design flow coefficient of 0.6. The blades are designed using the controlled diffusion concept. ${ }^{22}$ As can be seen from Fig. 1, the curvature of the suction surface is largest near the minimum pressure region to encourage maximum flow diffusion. The surface curvature upstream of the trailing edge is smaller, allowing less adverse pressure gradient, hence, easing the diffusion process for the boundary layer. Both the stator and rotor have the same chord, solidity, and trailing-edge thickness. Table 1 gives the relevant blade and compressor parameters used.

\section{Results and Discussions}

\section{A. Unsteady Velocities (Prior to Decomposition)}

Figures 4-7 present the unsteady flow, prior to decomposition, for the axial gap of $20 \% C$ at $t / T=0.0,0.2,0.4$, and 0.6 . The unsteady velocity is defined as the local time mean subtracted from the instantaneous velocity. These plots are helpful, not only to get an overall view of the flow in the gap

Table 1 Stator/rotor geometric and flow parameters used in calculations

\begin{tabular}{lcc}
\hline \hline & Stator & Rotor \\
\hline Chord & 1.00 & 1.00 \\
Solidity & 1.40 & 1.40 \\
Trailing-edge radius, \%C & $1.0 \%$ & $1.0 \%$ \\
Stagger, deg & 20.67 & -39.50 \\
Camber, deg & 48.0 & 35.0 \\
Inlet angle, $\beta$ 1, deg & 47.29 & 57.73 \\
Exit angle, $\beta$ 2, deg & 4.76 & 30.26 \\
Diffusion factor & 0.485 & 0.407 \\
Axial gap, \%C & 10,20, and 30 \\
\hline \hline
\end{tabular}




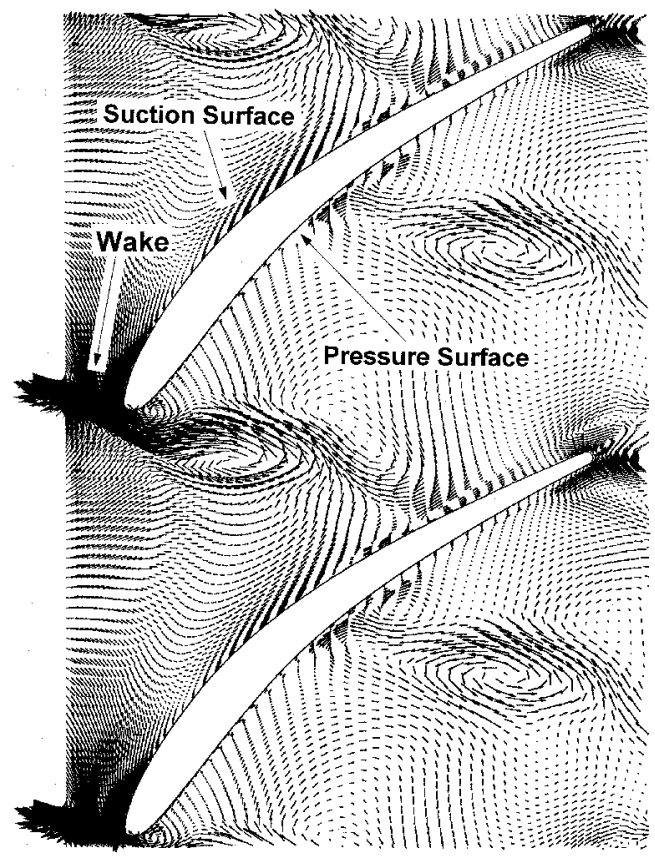

Fig. 4 Unsteady velocity vector within the rotor passage for the axial gap of $20 \% C$ at $t / T=0.0$.

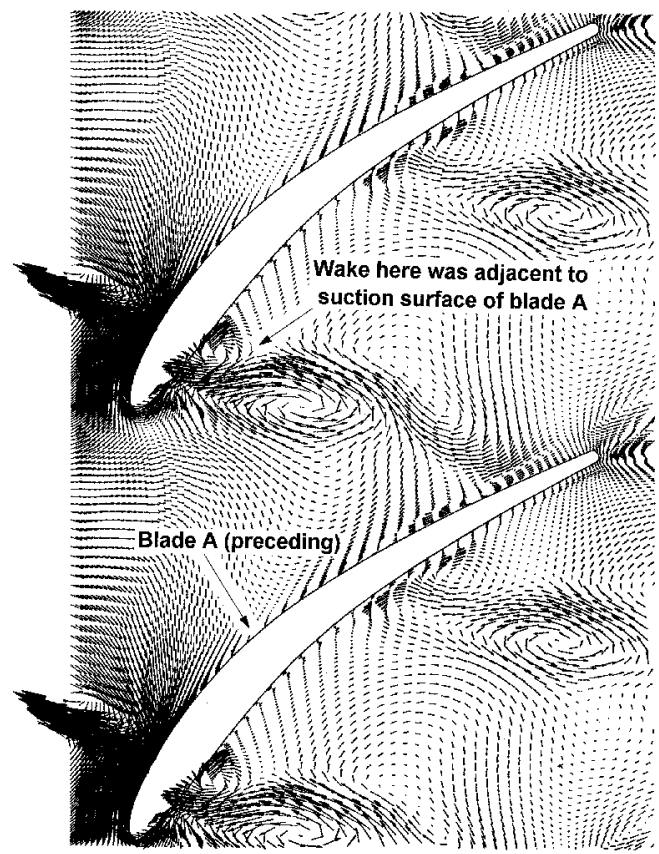

Fig. 5 Unsteady velocity vector within the rotor passage for the axial gap of $20 \% C$ at $t / T=0.2$.

and the blade-to-blade regions at a particular time instant, but they also show the flow process during an unsteady period.

At $t / T=0.0$, which corresponds to the stator/rotor relative position of Fig. 1, Fig. 4 shows the wake from the stator just meets the rotor leading edge, which creates a large instantaneous suction region on the suction surface near the nose. As will be shown later, this corresponds to a peak in the local pressure distribution, which is a dominant feature in the overall unsteady blade loading.

On the pressure surface, however, the local unsteady flow results in a jet-like structure toward the blade surface. This feature is better shown at $t / T=0.2$ (see Fig. 5) at about $10 \% C$ position. The visual effect of velocity vectors penetrating the blade surface indicates a large local unsteady flow immediately adjacent to and toward the wall. Comparing this structure for $t / T=0.2,0.4$, and 0.6 indicates that it weakens as the wake is convected along the pressure surface, which is also true for the flow structure adjacent to the suction surface.

There is a qualitative difference between the structure of the two wake portions separated by the blade. The portion of the wake adjacent to the suction side extends from the upstream stator trailing edge and terminates on the downstream rotor suction surface. The structure of this wake portion is mostly dominated by a velocity defect, with its unsteady characteristic influenced by the potential effect from the downstream blade row. ${ }^{12}$ However, an additional mechanism has a pronounced effect on the portion of the wake adjacent to the pressure surface. The fact that this wake portion extends from the forward region of the pressure surface to the aft of the suction surface of a preceding blade (see Fig. 5) provides a clue. Prior to its

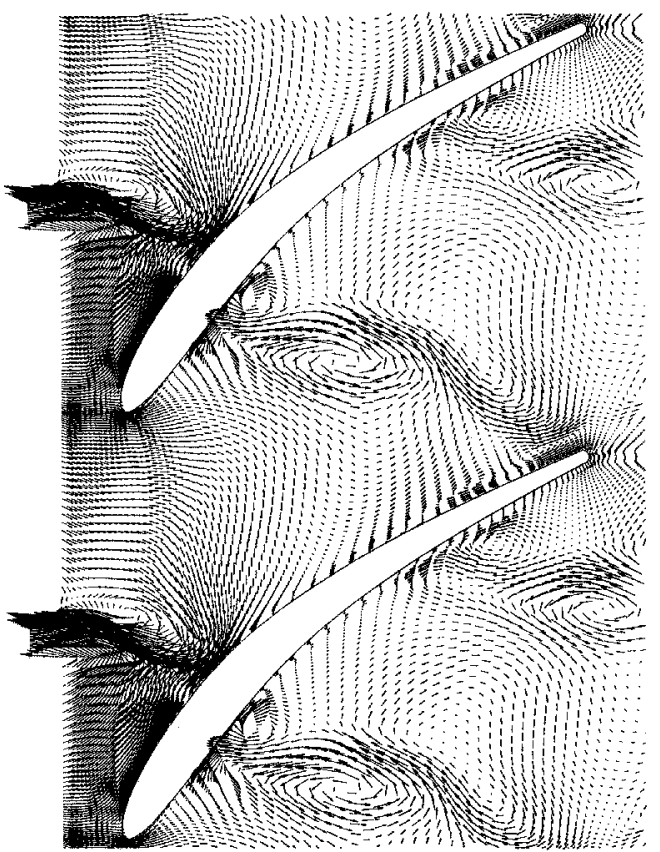

Fig. 6 Unsteady velocity vector within the rotor passage for the axial gap of $20 \% C$ at $t / T=0.4$.

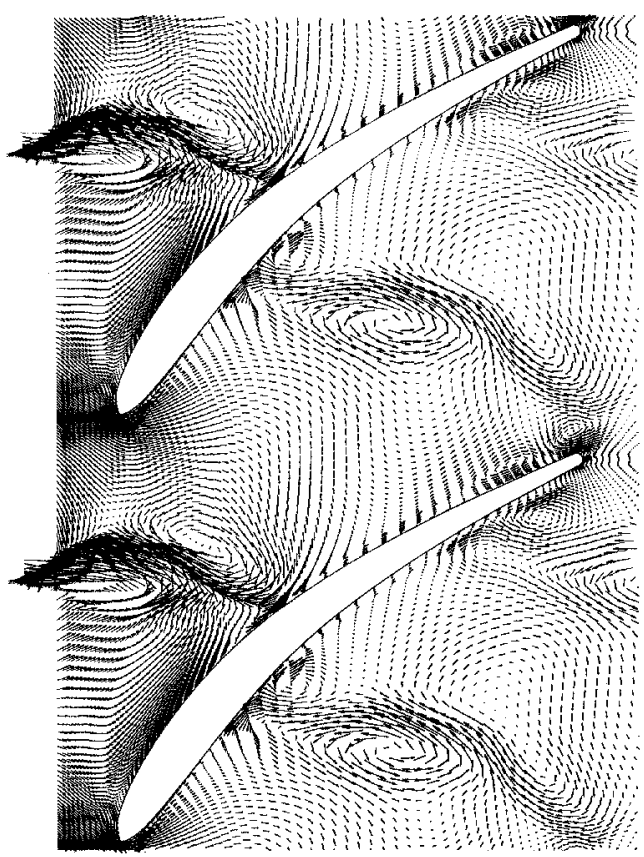

Fig. 7 Unsteady velocity vector within the rotor passage for the axial gap of $20 \% C$ at $t / T=0.6$ 
present location, this wake portion was actually that from the upstream stator trailing edge and ends on the suction surface of the preceding rotor blade. As this wake convects downstream it interacts with the rotor suction surface of the preceding rotor blade, and weakens the wake structure. As this wake arrives at the leading edge of the current blade, it is chopped and, thus, arrived at the present location adjacent to the pressure surface. In other words, unlike the wake portion adjacent to the suction surface, the wake portion adjacent to the pressure surface has already experienced interaction from the suction surface of the preceding rotor blade. This is believed to be the primary reason that the unsteadiness is much weaker on the pressure surface than the suction surface.

The rate of wake convection through the blade row deserves a comment. The wake takes approximately 1.4 unsteady periods before convecting past the suction surface of a particular blade, and takes two periods before convecting past the pressure surface. This is primarily because of the time mean circulation about a blade.

\section{B. Unsteady Pressure Distribution (Prior to Decomposition)}

The unsteady pressure along the rotor surface, prior to decomposition into vortical and potential effects, is presented in Figs. $8 \mathrm{a}$ and $8 \mathrm{~b}$. The chordwise distribution is distinct between the pressure and suction surfaces. On the pressure surface, apart from large variation near the leading-edge region, the unsteady pressure is essentially constant along the chord. However, the suction surface exhibits a much stronger chordwise pressure variation; there are local pressure maxima at various times.

Close examination of these peaks reveal a temporal variation similar to that of a convective flow phenomenon along the suction surface. This can be seen from a peak located near $s / S=0.2$ at $t / T=0.2$, which moves to $s / S=0.3$ at $t / T=0.4$, then $s / S=0.4$ at $t / T=0.6$, with a decaying amplitude. Unsteady vorticity contours reveal that these moving peaks correspond to the location of distinct vortices near the suction surface at that specific time.

The fundamental mechanism that induces these vortices is a nonlinear interaction between the moving wake and the local boundary layer, as demonstrated by Valkov and Tan. ${ }^{23}$ Using a spectral-element Navier-Stokes solver to study the unsteady flow arising from wake-stator interaction, they found that the unsteady flow over the suction surface of the stator blades is characterized by a moving row of vortical disturbances (B vortices) produced at the leading edge upon wake interception. The B vortices consist of boundary-layer fluid

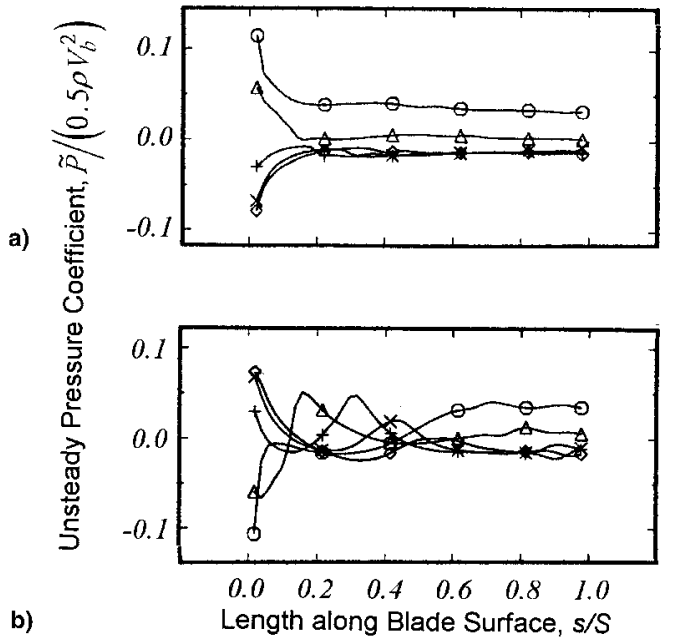

Fig. 8 Instantaneous unsteady pressure, prior to decomposition, on the rotor a) pressure and $b$ ) suction surfaces for the axial gap of $20 \% C . \circ, t / T=0.0 ; \triangle, t / T=0.2 ;+, t / T=0.4 ; \times, t / T=0.6$; and $\diamond, t / T=0.8$. a)

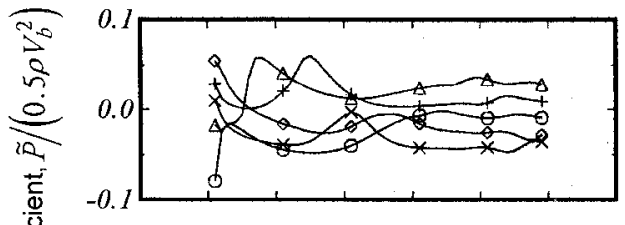

b)

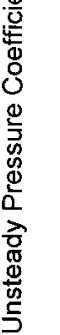

Fig. 9 Instantaneous unsteady pressure on the rotor suction surface, for the axial gap of $20 \% C$, from a) vortical and b) potential contributions. $\circ, t / T=0.0 ; \triangle, t / T=0.2 ;+, t / T=0.4 ; \times, t / T=0.6$; and $\diamond, t / T=0.8$.

that is distorted and lifted off the suction surface by the convective action of the wakes. These vortices are also identified in the unsteady vorticity calculation, it is believed that the gust response behavior on the suction surface must be caused by similar flow mechanisms in the boundary layer.

\section{Vortical and Potential Unsteady Pressure Distribution}

The unsteady pressure on the downstream rotor suction surface is decomposed into vortical and potential parts, and are shown in Figs. 9a and 9b, respectively, for an axial gap of $20 \% C$. The fact that the convective feature in the pressure signature, as described in Sec. VI.B for Fig. 8 b, is caused by flow phenomenon associated with the wake, is clearly shown in the vortical contribution of Fig. 9a. The potential contribution exhibits a constant phase excitation about the suction surface, except very near the leading-edge region.

\section{Unsteady Force Caused by Vortical and Potential Contributions}

The decomposed unsteady axial and tangential force are shown in Fig. 10 for the three axial gaps calculated. The three lines drawn on each plot are obtained from the integrated unsteady pressure because of either a vortical and potential disturbance or the sum of the two (note that the zero in the ordinate is offset).

First, all unsteady flow interaction increases as the axial gap decreases. The potential contribution is essentially zero for $30 \%$ gap, whereas the vortical contribution persists over a large gap range.

Consider the axial force at the axial gap of $20 \% C$. At $t / T=$ 0.05 , the total unsteady force is dominated by the vortical contribution, which is an extremum. The reason for this extremum is because of large pressure differences near the leading edge as the wake just arrives at the rotor leading edge (see Fig. 4). This can be inferred by Fig. 8, which shows a large unsteady loading near the leading edge. (Although the unsteady pressure in Fig. 8 is not yet decomposed, the potential contribution is much smaller than the vortical part, as can be seen from Fig. 10.)

Similarly, at $10 \% C$ axial gap, the total unsteady force is dominated by the vortical contribution at $t / T=0.0$. For $0.3<$ $t / T<0.9$, both vortical and potential parts are mutually important in determining the total unsteady force. This has a profound implication to minimize the total unsteady force. Perhaps one can tune either the vortical or potential contribution, or both, by altering the fundamental mechanism involved. This is currently under further investigation.

The vortical contribution in the blade force reaches an extremum when the upstream wake impinges upon the down- 


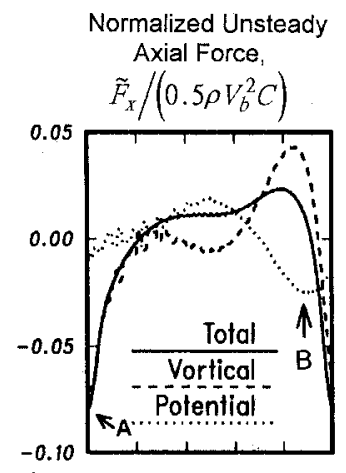

a)

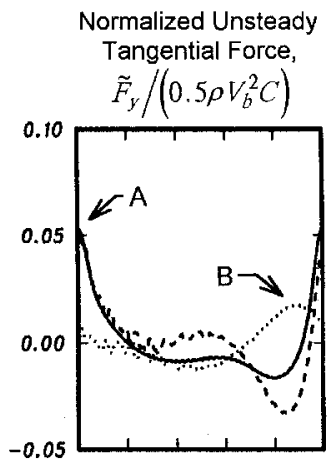

d)

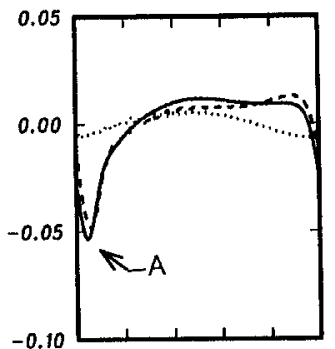

b)

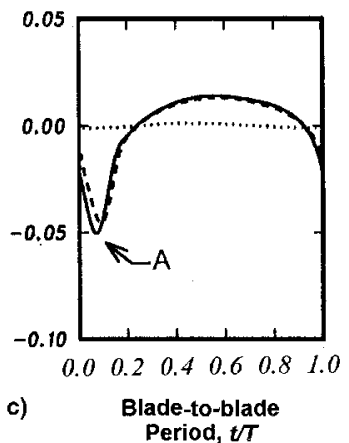

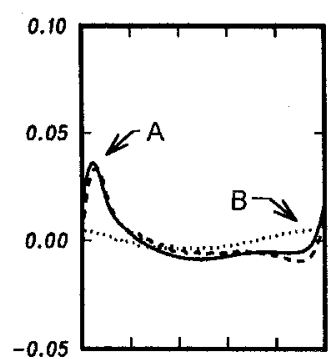

e)

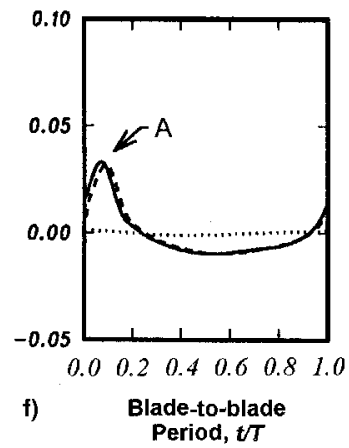

VII. Concluding Remarks

The unsteady pressure on a rotor blade surface because of flow unsteadiness has been decomposed into vortical and potential contributions. The effect of blade row axial spacing on the decomposition for a stator/rotor configuration near the design point is studied numerically. Major results are as follows:

1) Three methods are outlined to perform such decomposition, based on the control volume approach, direct unsteady pressure calculation from the panel and Navier-Stokes codes, and the use of the Navier-Stokes code with either disturbance as the inlet boundary condition. The latter method is demonstrated in this work.

2) The extremum for the unsteady force caused by vortical contribution occurs near the instant when the wake from the upstream blade arrives at the leading edge of the downstream blade. This causes a large local pressure difference near the leading edge.

3) The extremum for the unsteady force caused by potential contribution occurs when the trailing edge of the upstream stator vane is closest to the leading edge of the downstream blade.

4) Unlike the wake portion adjacent to the suction surface, the wake portion adjacent to the pressure surface has already experienced interaction with the suction surface of the preceding blade. This is believed to be the primary reason that the wake structure, and its associated unsteadiness, is much weaker on the pressure surface than the suction surface.

5) A temporal variation of unsteady pressure exists similar to that of a convective flow phenomenon along the suction surface of the downstream blade. This is because of a nonlinear interaction between the moving wake and the local boundary layer. $^{23}$

\section{Acknowledgment}

The authors are grateful for the financial support of the National Science Council, Republic of China, Grant NSC 842212-E-002-007.

\section{References}

${ }^{1} A G A R D$ Manual on Aeroelasticity in Axial-Flow Turbomachines, Unsteady Turbomachinery Aerodynamics, Vol. 1, edited by M. F. Platzer and F. O. Carta, AGARD-AG-298, March 1987.

${ }^{2}$ Kielb, R. E., and Chiang, H. D., "Recent Advancements in Turbomachinery Forced Response Analyses," AIAA Paper 92-0012, Jan. 1992.

ZVerdon, J. M., "Review of Unsteady Aerodynamic Methods for Turbomachinery Aeroelastic and Aeroacoustic Applications," AIAA Journal, Vol. 31, No. 2, 1993, pp. 235-250.

${ }^{4}$ Verdon, J. M., and Hall, K. C., "Development of a Linearized Unsteady Aerodynamic Analysis for Cascade Gust Response Predictions," NASA Contract NAS3-25425, 1990.

5Hall, K., and Crawley, E., "Calculation of Unsteady Flows in Turbomachinery Using the Linearized Euler Equations," AIAA Journal, Vol. 27, No. 6, 1989, pp. 777-787.

Giles, M. B., "Calculation of Unsteady Wake/Rotor Interaction," Journal of Propulsion and Power, Vol. 4, No. 4, 1988, pp. 356-362.

Rai, M. M., "Navier-Stokes Simulations of Rotor/Stator Interaction Using Patched and Overlaid Grids," Journal of Propulsion and Power, Vol. 3, No. 5, 1987, pp. 387-396.

Rai, M. M., and Madavan, N. K., "Multi-Airfoil Navier-Stokes Simulations of Turbine Rotor/Stator Interaction," Journal of Turbomachinery, Vol. 112, No. 3, 1990, pp. 377-384.

${ }^{9}$ Manwaring, S. R., and Wisler, D. C., "Unsteady Aerodynamics and Gust Response in Compressors and Turbines," Journal of Turbomachinery, Vol. 115, No. 4, 1993, pp. 724-740.

${ }^{10}$ Henderson, G., and Fleeter, S., "Forcing Function Effects on Unsteady Aerodynamic Gust Response: Part 1-Forcing Functions," Journal of Turbomachinery, Vol. 115, No. 4, 1993, pp. 741-750.

${ }^{11}$ Henderson, G., and Fleeter, S., "Forcing Function Effects on Unsteady Aerodynamic Gust Response: Part 2-Low Solidity Airfoil Row Response," Journal of Turbomachinery, Vol. 115, No. 4, 1993, pp. $751-761$.

${ }^{12}$ Chung, M. H., and Wo, A. M., "Navier-Stokes and Potential Calthe flow can be regarded as incompressible. 
culations of Axial Spacing Effect on Vortical and Potential Disturbances and Gust Response in an Axial Compressor," Journal of Turbomachinery (to be published).

${ }^{13}$ Chung, M. H., "Numerical Study of Vortical and Potential Disturbances and Gust Response in an Axial Compressor," Ph.D. Dissertation, Inst. of Applied Mechanics, National Taiwan Univ., Taipei, Taiwan, ROC, 1995.

${ }^{14}$ Morse, A. P., "Application of a Low Reynolds Number Turbulence Model to High-Speed Rotating Cavity Flows," Journal of Turbomachinery, Vol. 113, No. 1, 1991, pp. 98-105.

${ }^{15}$ Patankar, S. V., and Spalding, D. B., "A Calculation Procedure for Heat, Mass and Momentum Transfer in Three-Dimensional Parabolic Flows," International Journal of Heat and Mass Transfer, Vol. 15, 1972, pp. 1787- 1797.

${ }^{16}$ Leonard, B. P., "A Stable and Accurate Convective Modeling Procedure Based on Quadratic Upstream Interpolation," Computer Methods in Applied Mechanics and Engineering, Vol. 12, 1979, pp. 5998.

${ }^{17}$ Kuethe, A. M., and Chow, C. Y., "Aerodynamic Characteristics of Airfoils," Foundations of Aerodynamics, 4th ed., Wiley, New York, 1986, pp. 106-137, Chap. 5.
${ }^{1}$ Lamb, H., "Vortex Motion," Hydrodynamics, 6th ed., Dover, New York, 1945, pp. 202-249, Chap. VII.

${ }^{19}$ Basu, B. C., and Hancock, G. J., "The Unsteady Motion of a Two-Dimensional Airfoil in Incompressible Inviscid Flow," Journal of Fluid Mechanics, Vol. 87, Pt. 1, 1978, pp. 159-178.

${ }^{20}$ Sears, W. R., "Unsteady Motion of Airfoils with Boundary-Layer Separation," AIAA Journal, Vol. 14, No. 2, 1976, pp. 216-220.

${ }^{21}$ Feiereisen, J. M., Montgomery, M. D., and Fleeter, S., "Unsteady Aerodynamic Forcing Functions: A Comparison Between Linear Theory and Experiment," American Society of Mechanical Engineers, Paper 93-GT-141, 1993.

${ }^{22}$ Hobbs, D. E., and Weingold, H. D., "Development of Controlled Diffusion Aerofoils for Multistage Compressor Applications," Journal of Engineering for Gas Turbine and Power, Vol. 106, 1984, pp. 271 -278 .

${ }^{23}$ Valkov, T., and Tan, C. S., "Control of the Unsteady Flow in a Stator Blade Row Interacting with Upstream Moving Wakes," Journal of Turbomachinery, Vol. 117, No. 1, 1995, pp. 97-105.

${ }^{24}$ Goldstein, M. E., "Unsteady Vortical and Entropic Distortions of Potential Flows Around Arbitrary Obstacles," Journal of Fluid Mechanics, Vol. 89, Pt. 3, 1978, pp. 433-468. 